Revue internationale de l'économie sociale

Recma

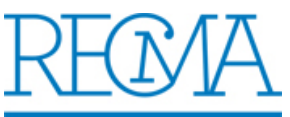

\title{
Syndicalisme et ESS : l'expérience québécoise
}

\section{Yvon Poirier}

Numéro 322, octobre 2011

URI : https://id.erudit.org/iderudit/1020727ar

DOI : https://doi.org/10.7202/1020727ar

Aller au sommaire du numéro

\section{Éditeur(s)}

Association Recma

\section{ISSN}

1626-1682 (imprimé)

2261-2599 (numérique)

Découvrir la revue

\section{Citer cet article}

Poirier, Y. (2011). Syndicalisme et ESS : l'expérience québécoise. Revue internationale de l'économie sociale, (322), 16-22.

https://doi.org/10.7202/1020727ar d'utilisation que vous pouvez consulter en ligne.

https://apropos.erudit.org/fr/usagers/politique-dutilisation/ 


\title{
SYNDICALISME ET ESS: L'EXPÉRIENCE QUÉBÉCOISE
}

\author{
par Yvon Poirier*
}

\footnotetext{
* Président du comité international du Réseau canadien de développement économique communautaire (RCDEC), membre du Réseau intercontinental de promotion de l'économie sociale et solidaire (Ripess).
}

- es syndicats de travailleurs du Québec soutiennent l'économie sociale et solidaire (ESS). Ils ont créé des fonds d'investissement et des caisses d'épargne et de crédit. Une organisation syndicale en particulier, la Confédération des syndicats nationaux (CSN), a été à l'avant-garde de ces activités. L'auteur, militant à la Confédération des syndicats nationaux (CSN) au niveau local, régional et national de 1968 à 1994, aborde ici la question du rôle des syndicats dans le renforcement de l'économie solidaire ${ }^{(1)}$.

\section{Les syndicats au Québec: bref aperçu historique}

(1) Ce texte est une version remaniée d'un article publié dans Solidarity economy l: Building alternatives for the people and the planet, Kawano E., Neal Masterton T. and Teller-Elsberg J. Center for Popular Economics, Amherst MA, USA, June 2010, pp. 99-113.

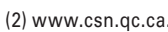

Au Québec, le syndicalisme s’est développé selon deux courants distincts. Le premier émane des syndicats américains, comme les Chevaliers du travail, qui au $\mathrm{XIX}^{\mathrm{e}}$ siècle ont été à l'origine de nombreuses initiatives au Canada et dans la province de Québec. Il s'agit d'une situation originale, car dans la plupart des pays, les syndicats sont des organisations nationales affiliées à des fédérations internationales. Or au Canada, dans la première partie du Xx ${ }^{\mathrm{e}}$ siècle, la plupart des syndicats étaient affiliés à des syndicats américains. Aujourd'hui encore, de nombreux travailleurs canadiens sont membres de syndicats nord-américains; ainsi, les Métallos, section québécoise des United Steelworkers, constituent le gros syndicat du Québec. Au fil du temps cependant, certains membres sont devenus des syndicats indépendants, à l'instar des Travailleurs canadiens de l'automobile, qui se sont séparés en 1984 de l'United Auto Workers des Etats-Unis.

Le deuxième courant, d'inspiration principalement européenne et catholique, visait l'indépendance à l'égard des syndicats internationaux, notamment américains. Les syndicats catholiques ont été organisés à la suite de la promulgation de l'encyclique Rerum Novarum (1891), par laquelle le pape Léon XIII condamnait la misère ouvrière. Comme l'Eglise catholique a occupé une place très importante dans la société québécoise jusqu’en 1960, elle a exercé un leadership important dans la création des syndicats. En 1921, les différents syndicats catholiques de la province ont créé la Confédération des travailleurs catholiques du Canada. Cette organisation est devenue laïque en 1960, sous le nom de Confédération des syndicats nationaux $(\mathrm{CSN})^{(2)}$.

Depuis plus de trente ans, le Québec affiche le taux de syndicalisation le plus élevé d'Amérique du Nord, soit environ $40 \%$, contre $14 \%$ aux 
(3) http://reco.concordia.ca/pdf/ WP\%20Lamarche06.pdf.
Etats-Unis. Cette situation est d'autant plus remarquable que ce taux a baissé dans la plupart des pays de l'OCDE.

La nature de la société québécoise ne suffit pas à expliquer ce phénomène: les dirigeants syndicalistes ont été inspirés par la social-démocratie scandinave et allemande, qui considère les syndicats comme des partenaires sociaux au même titre que le gouvernement et les employeurs. De plus, loin de restreindre leurs activités à la négociation collective et à la protection des droits des travailleurs, les syndicats du Québec ont étendu leurs activités à la plupart des questions sociales, comme l'égalité des sexes, la paix, la pauvreté, la réforme électorale, la santé et l'éducation, l'égalité des droits pour les gays et lesbiennes, l'environnement, etc.

Comme le dit François Lamarche, l'appui à l'économie sociale et solidaire est également un facteur clé: "Du point de vue syndical, l'économie sociale et solidaire n'est pas seulement perçue comme un secteur d'activité campé quelque part entre l'économie publique et l'économie privée. Elle est surtout vue comme participant ou devant participer à un mouvement de transformation sociale ${ }^{(3)}$."

\section{La CSN, un acteur clé de l'ESS}

La CSN a été au cœur de nombreuses initiatives d'économie sociale et solidaire, ce qui s'explique en partie par la structure organisationnelle de ce syndicat. La CSN est organisée autour de trois piliers: la confédération elle-même, les fédérations et les conseils centraux (dans les villes ou les régions). Les conseils centraux sont semblables aux conseils du travail en place au sein du Congrès du travail du Canada (CTC) ou de l'American Federation of Labor and Congress of Industrial Organizations (AFL-CIO) aux Etats-Unis. Toutefois, dans le cas de la CSN, cette triple affiliation est obligatoire pour les syndicats locaux, alors qu'elle est facultative dans le cas de la CTC. Les syndicats de la CSN disposent donc de ressources financières plus importantes, leur permettant de s'engager activement au niveau local sur de nombreux enjeux sociaux. En plus de cet avantage en termes de capacité d'action locale, la CSN a été, dans les années 60, un acteur important dans le processus de transformation de la société québécoise, en mettant en avant une vision progressiste de la société.

Lors du congrès de 1968, la CSN a adopté la stratégie du "deuxième front ", le premier étant la négociation collective et la défense des droits des travailleurs. Dans un document interne, ce choix est présenté ainsi: "Ce "deuxième front" veut lier les luttes syndicales aux luttes populaires de l'époque et propose comme objectif d'élargir l'action syndicale aux domaines de la consommation et des conditions de vie. C'est ainsi que des militants syndicaux s'impliquent dans la création de coops d'alimentation, de comptoirs alimentaires, de coopératives de logement. La CSN appuie activement l'action des comités de citoyens dans le domaine de la santé, particulièrement 
(4) Centres locaux de services communautaires. Les cliniques populaires ont été intégrées au système de santé publique au début des années 70 (NDLR) (5) Centres de la petite enfance. Associations ou coopératives, soutenues par le gouvernement depuis 1997, gérées par un conseil d'administration composé d'au moins deux tiers de parents utilisateurs des services de gardes (NDLR).

(6) La CSN et l'économie sociale, 2006. la création de cliniques médicalespopulaires (lesquelles deviendrontparla suite les premiers $\left.C L S C^{(4)}\right)$. C'est aussi à cette époque que des militantes de la CSN sassocient aux groupes de femmes et de parents pour la création de garderies populaires (lesquelles constitueront la base du futur réseau des $\left.C P E^{(5)}\right)^{(6)}$. " Cette perspective a inspiré d'autres initiatives. Par exemple, les ambulanciers syndiqués se sont constitués en une coopérative de travail qui assure le service public de transport ambulancier. Plus récemment, la CSN a donné un appui formel aux initiatives de commerce équitable.

Aux niveaux municipal, régional et provincial, des représentants de la CSN agissent régulièrement comme membres de conseil d'administration d'organismes de développement tels que les corporations de développement économique communautaire (CDEC) ou les centres locaux de développement (CLD).

\section{Coopératives et organismes sans but lucratif}

Au Québec, le secteur des coopératives est l'un des plus développés parmi les pays de l'OCDE, en particulier dans le secteur financier. Bien que certaines coopératives aient existé auparavant, le mouvement coopératif moderne a pris son essor avec Alphonse Desjardins, qui a fondé la première caisse populaire en 1897. Avec l'aide de l'Eglise, ce modèle s'est étendu à toute la province et a ouvert, pour la première fois, un accès aux activités bancaires pour la communauté francophone.

Le Mouvement Desjardins est devenu la plus importante institution financière de la province de Québec et l'une des plus importantes dans le monde avec des actifs, dépassant 152 milliards de dollars (108 millions d'euros) et 5,8 millions de membres. Au total, plus de $80 \%$ de la population adulte de la province est membre d'une caisse populaire.

Depuis 1930, des coopératives ont été créées dans plusieurs secteurs. Plusieurs petites agglomérations comptent une caisse populaire, une épicerie coopérative et une coopérative agricole. Aujourd'hui, plus de 3000 coopératives non financières réalisent un chiffre d'affaires annuel de 10 milliards de dollars (7,14 milliards d'euros). Elles opèrent dans tous les secteurs: l'agriculture et la pêche, l'alimentation, la sylviculture, les coopératives de travail, l'aide à domicile, le logement et les services funéraires. Les données officielles montrent que les coopératives ont un taux de survie beaucoup plus élevé que les entreprises privées: après trois ans, il est de $75 \%$ pour les premières, contre $48 \%$ pour les secondes; après dix ans, ce taux est respectivement de 40 et $20 \%$. Des modifications législatives récentes permettent la création de coopératives de solidarité qui réunissent diverses catégories de membres (producteurs, travailleurs, usagers, partenaires) à l'image des coopératives sociales italiennes.

Au cours des trente dernières années, de nombreuses entreprises d'économie sociale ont également été créées en utilisant les dispositions de la partie III de la loi des compagnies, qui permet de fonder des organismes sans but lucratif (OSBL). Certaines de ces associations sans but lucratif ont organisé leurs activités de façon à générer des revenus. D’autres 
utilisent ce statut plutôt que de recourir à la loi sur les coopératives, qui est plus contraignante.

\section{Bâtir l'économie solidaire : de 1970 à 1995}

(7) Bulletin international de développement local durable, $\mathrm{n}^{\circ} 39$, juin 2007, Yvon Poirier. A lire sur http://developmentlocal. blogspot.com.
Suite aux bouleversements économiques et sociaux survenus entre 1970 et 1995, les syndicats ont poussé les gouvernements à adopter des mesures de soutien à l'emploi et même revendiqué une solution globale par des lois assurant le plein emploi. Tout en luttant pour l'adoption de telles mesures, les syndicats au Québec ont compris qu'ils devaient également eux-mêmes prendre des initiatives.

\section{Caisse d'économie solidaire Desjardins: une caisse populaire innovante}

En 1971, des militants CSN de la ville de Québec décident de créer une nouvelle caisse populaire avec une mission innovante. Il existait déjà une caisse traditionnelle dont l'activité principale était de fournir les services d'épargne et de crédit aux particuliers. La nouvelle caisse a opté dès le départ pour une approche plus collective. Au lieu d'avoir le meilleur taux d'intérêt sur les dépôts, on choisit de mettre en place des services collectifs comme l'achat en groupe de mazout pour le chauffage domestique. Les syndicats membres et les syndiqués membres individuels sont également convenus de placer certaines épargnes dans des comptes sans intérêts, afin que ces fonds puissent être prêtés à un très faible taux pour la construction de coopératives d'habitation et sans intérêts pour les travailleurs en grève. La Caisse d'économie des travailleurs réunis de Québec a rejoint le Mouvement Desjardins en 1979, en partie pour avoir accès aux services informatiques comme le dépôt direct. Jusqu’en 2002, la caisse a fusionné avec d'autres caisses de travailleurs et pris le nom de Caisse d'économie solidaire Desjardins. Au fil des ans, d'autres syndicats, tels ceux des enseignants et des syndicats du secteur public, sont devenus membres. Même si elle n'est que l'une des cinq cents caisses du Mouvement Desjardins, elle a gardé sa philosophie initiale de mise en commun de l'épargne des travailleurs pour améliorer la qualité de vie et travailler ensemble à construire une économie solidaire ${ }^{(7)}$.

En 2011, la caisse est au cœur de l'économie sociale et solidaire du Québec et elle est reconnue internationalement comme étant à la pointe de la construction d'une économie citoyenne. Il convient de souligner sa remarquable capacité à remplir sa mission en restant fidèle à ses valeurs d'origine sans négliger toutes les obligations et règles qu'elle rencontre au même titre que toutes les banques.

\section{Les fonds de travailleurs: une approche novatrice}

Au Canada, des régimes de retraite existent dans le secteur public et dans les grandes entreprises. Les professions libérales et la plupart des 
PME n'ont pas de régime de retraite collectif. Pour encourager l'épargne retraite, les gouvernements ont mis en place des régimes enregistrés d'épargne retraite (REER). Tous les investissements dans ces fonds sont déductibles des impôts à $100 \%$. L'impôt est payé seulement quand une personne y retire de l'argent.

En 1984, la Fédération des travailleurs du Québec (FTQ) a demandé au gouvernement du Québec les moyens de mettre en place un fonds de travailleurs à partir de l'épargne retraite. Une partie du capital du fonds peut être investie dans l'économie du Québec afin de créer et de sauvegarder des emplois, tout en incitant les travailleurs à épargner pour la retraite. Il s'agit d'une initiative très intéressante, car la priorité des fonds de pension est généralement de maximiser la croissance du capital. Comme la création d'emplois pouvait être un investissement relativement risqué pour l'épargne retraite, un crédit d'impôt de $30 \%$ (provenant à $15 \%$ de la province et à $15 \%$ du fédéral) a été accordé à cette mesure. Le fonds, baptisé Fonds de solidarité des travailleurs et travailleuses du Québec (FSTQ), a été créé par une loi en 1984 et est rapidement devenu très populaire. Disposant d'environ 8 milliards de dollars (mai 2011), c'est l'un des plus grands fonds d'investissement de la province. Il est géré par un conseil d'administration issu principalement de la FTQ. La loi précise que sa mission est d'investir dans les entreprises québécoises et de leur fournir des services en vue de contribuer à leur développement. Le FSTQ n'investit pas dans une entreprise qui n'est pas syndiquée.

En 1995, le gouvernement a accepté une proposition de la CSN à l'effet de créer un deuxième fonds de travailleurs, nommé Fondaction. A la différence du FSTQ, sa mission est de soutenir le développement de coopératives et la création d'emplois et d'investir dans des entreprises dotées d'une organisation du travail impliquant les travailleurs et soucieuses de l'environnement. La mission de Fondaction est donc plus orientée vers l'économie sociale que celle du FSTQ, mais le mode d'opération, les avantages fiscaux et l'obligation d'investir au moins $60 \%$ dans les entreprises de la province de Québec sont similaires. Le conseil d'administration est composé principalement de membres de la CSN. En mai 2011, l'épargne accumulée dans Fondaction était d'environ 850 millions de dollars (595 millions d'euros).

\section{Bâtir l'économie solidaire: de 1996 à aujourd'hui}

La marche des femmes "Du pain et des roses", en juin 1995, a constitué un événement historique au Québec. Convergeant vers la capitale, le Mouvement des femmes et ses alliés réclamaient une action du gouvernement concernant les enjeux économiques et sociaux auxquels les femmes sont confrontées, comme la pauvreté et les faibles revenus. Cette marche a donné naissance à une marche mondiale des femmes qui s'est rendue à l'ONU en 2009 et a suscité la création de l'organisation internationale du même nom. 
En 1996, le gouvernement du Québec a organisé un sommet avec les différents partenaires sociaux, y compris le Mouvement des femmes. Face au taux élevé de chômage et à l'envolée de la dette publique, le gouvernement voulait réduire à zéro le déficit budgétaire. Le Premier ministre Lucien Bouchard, à la tête d'un gouvernement du Parti québécois (parti nationaliste social-démocrate), a appelé un sommet socio-économique où, pour la première fois dans l'histoire du Québec, des organisations sociales et communautaires ont été invitées en tant que partenaires sociaux au même titre que le gouvernement, les entreprises et les syndicats. Différents chantiers de travail ont été créés, dont un sur l'économie sociale. Lors de la réunion finale du sommet, en octobre 1996, tous les partenaires sociaux ont adopté l'économie sociale comme stratégie pour créer des emplois et lutter contre la pauvreté. Les syndicats ont alors renoncé à des augmentations de salaire en contrepartie de l'engagement du gouvernement et du secteur capitaliste privé d'appuyer l'économie sociale. Certaines grandes sociétés ont même accepté de verser quelques millions de dollars à un fonds de capital risque, afin de soutenir le démarrage d'entreprises sans but lucratif (OSBL). Elles estimaient que leur contribution au Réseau d'investissement social Québec (Risq) était une aumône, mais aujourd'hui la majorité du capital est toujours disponible, ce qui prouve que les entreprises d'économie sociale peuvent devenir viables. Seule note discordante, certains mouvements sociaux ont considéré que cette approche était trop réformiste et certains secteurs du mouvement syndical craignaient que le gouvernement ne privatise des services publics. Cette crainte des syndicalistes s’est en grande partie dissipée au fil des ans.

\section{Le Chantier de l'économie sociale du Québec et la CSN}

Dès le début, la CSN a décidé de rejoindre le Chantier de l'économie sociale (www.chantier.qc.ca) du Québec, mis en place après le Sommet de l'économie et de l'emploi de 1996 avec le soutien du gouvernement pour développer les programmes. Aux côtés de la CSN et d'autres syndicats, de nombreux mouvements sociaux et organismes de développement se sont joints au chantier. Par exemple, sont devenus membres le Mouvement des femmes, des groupes environnementaux, des organismes de développement local, ainsi que différentes organisations directement concernées comme l'Association québécoise des centres de la petite enfance. En somme, le chantier est devenu un réseau de réseaux d'économie sociale au Québec, à l'exception de celui du secteur coopératif, qui en général préfère travailler sur ses propres bases (www.coopquebec.ca). Certaines coopératives, tout en demeurant membres du réseau des coopératives, sont également membres du chantier.

Peu de temps après le sommet de 1996, un accord est intervenu entre la CSN et le chantier pour que celui-ci ne fasse pas la promotion et ni soutienne en aucune façon les entreprises d'économie sociale dont la création entraînerait, directement ou indirectement, la perte d'emplois dans le secteur public. En particulier, il a été convenu que les soins médicaux 
(comme les tests de pression sanguine ou les injections) ne seraient pas dans le mandat des organisations sans but lucratif d'aide domestique.

La CSN est aussi indirectement engagée dans une autre initiative nationale. En 2007, le chantier a obtenu la création d'un fonds d'investissement dans les entreprises d'économie, la Fiducie du chantier. Celle-ci a été mise en place grâce aux contributions du gouvernement fédéral (16,2 millions d'euros), du gouvernement du Québec (7 millions d'euros), du FSTQ (8,4 millions d'euros) et de Fondaction (5,6 millions d'euros). Il est intéressant de noter que les deux fonds de travailleurs ont investi près de $40 \%$ du total.

En 2006, la CSN, qui est également membre de la Confédération syndicale internationale (CSI), adopte "une vision inclusive de l'économie sociale et solidaire " comme alternative à la mondialisation néolibérale. En partenariat avec les outils collectifs tels que Fondaction ou la Caisse d'économie, elle crée Développement solidaire international (DSI) pour soutenir le travail de coopération internationale. Au Brésil, par exemple, il existe un accord de partenariat officiel avec la Centrale unique des travailleurs (CUT) pour développer des outils financiers semblables à ceux que la CSN a créés au Québec.

Les défis auxquels sont confrontés les syndicats et l'économie solidaire restent importants. Les relations entre les syndicats et le mouvement coopératif ne sont pas toujours faciles. Certaines coopératives tentent d'empêcher la syndicalisation des travailleurs. D’une façon générale, dans les grandes organisations, seule une minorité de travailleurs est syndiquée. Le taux de syndicalisation est quasi nul chez les employés de la plupart des caisses populaires. Les syndicats eux-mêmes sont confrontés aux attaques du néolibéralisme, comme dans bon nombre de pays où les travailleurs se voient contraints d'abaisser leurs exigences pour demeurer compétitifs. Très souvent, le maintien des emplois est en jeu. Syndicats et coopératives ont été la première ligne de défense organisée par les travailleurs au XIX ${ }^{\mathrm{e}}$ siècle pour lutter contre le capitalisme sauvage et proposer des alternatives. Non seulement ils doivent travailler main dans la main, mais ils doivent s'unir avec les autres mouvements sociaux pour résister au néolibéralisme et créer une société qui place l'économie au service de l'humanité et de la planète. 